\title{
Ethische Entscheidungen in zunehmend ökonomisierten Krankenhäusern
}

\author{
Eine Untersuchung zum ethischen Misstrauen in Verweildauerentscheidungen \\ bei Studierenden und ÄrztInnen unterschiedlicher Positionen
}

\author{
Ibrahim Alkatout (D) - Micha Strack · Nicolai Maass · Margarete Boos · Norbert Hopf \\ Eingegangen: 25. November 2019 / Angenommen: 13. Februar 2020 / Online publiziert: 28. Februar 2020 \\ (C) Der/die Autor(en) 2020
}

Zusammenfassung Werden durch die Ökonomisierung des deutschen Gesundheitssystems medizinische Werte in den Hintergrund gedrängt? Im Rahmen einer Vignettenstudie wurde der Fall einer Verlängerung der Verweildauer einer älteren, noch nicht gänzlich genesenen Patientin vorgestellt. Zunächst sollte über die Verweildauer entschieden werden. Ergänzend hierzu wurde die Relevanz der medizinischen Richtigkeit, Einfühlvermögen für die Patientin und Identifikation mit dem Klinikum erhoben. Die Teilnehmenden $(N=1239)$ schreiben den Entscheidern die medizinische Korrektheit als wichtigstes Kriterium zu, gefolgt vom Einfühlungsvermögen. Je universalistischer die Werte der befragten Person, umso eher befürwortet sie ein verlängertes Verweilen der Patientin im Krankenhaus. Je sicherheitsorientierter, je weniger tolerant und weniger prosozial, umso eher würde vorzeitig entlassen. Insbesondere fürchten Studierende eine verfrühte Entlassung durch die Entscheidungen des Chefarztes. Dies ist jedoch

Zusatzmaterial online Zusätzliche Informationen sind in der Online-Version dieses Artikels (https://doi.org/10.1007/ s10354-020-00742-5) enthalten.

Prof. Dr. med. I. Alkatout, M.A. MaHM ( $\bowtie)$.

Prof. Dr. med. N. Maass

Campus Kiel, Klinik für Gynäkologie und Geburtshilfe, Kiel School of Gynecological Endoscopoy, Universitätsklinikum Schleswig-Holstein, Arnold-Heller-Straße 3 / Haus

1B, 24105 Kiel, Deutschland

kiel.school@uksh.de

PD Dr. rer-nat. M. Strack • Prof. Dr. phil. M. Boos Georg-Elias-Müller-Institut für Psychologie,

Georg-August-Universität Göttingen,

Goßlerstraße 14, 37073 Göttingen, Deutschland

Dipl.-Kfm. N. Hopf

Hochschule für Agrar- und Umweltpädagogik,

Angermayergasse 1, 1130 Wien, Österreich als unbegründet zu werten. Aus den Zuschreibungen lässt sich interpretieren, dass sich ÄrztInnen im Laufe ihrer Ausbildung eine medizinethisch basierte, deontologisch fundierte Entscheidungsautonomie aneignen, die sich im Einzelfall auch aktuellen Regeln widersetzen kann.

Schlüsselwörter Ökonomisierung · Ethik · Werte · Krankenhausverweildauer · Fragebogenstudie

Ethical decision-making in the face of increasing economization of hospitals

A study on ethical mistrust in decisions taken on the length of hospital stay among students and doctors

Summary Are medical values receding in importance because of economization of the German health system? Within the frame of a vignette study, a case is presented based on prolongation of the hospitalization of an elderly and not entirely recovered patient. All respondents of the questionnaire predicted the relevance of decision criteria, such as medical accuracy, empathy towards the patient, and identification with the hospital. Participants $(N=1,239)$ believe that decision-makers view medical accuracy as the most important criterion, followed by empathy. The more the respondent had universalistic values, the more likely the person was to favor an extended hospitalization. The more security-oriented and less pro-social the respondent, the more likely the person was to support an early discharge. It can be concluded that in the course of their training doctors acquire their grounded deontological-ethical decision-making autonomy, which may in some cases contradict existing regulations. 
Keywords Economization · Ethics · Values · Period of hospitalization · Survey

\section{Einleitung}

Ärztliches Handeln ist heutzutage Diener zweier Herren. Zuvorderst steht es in der Verantwortung gegenüber den Patientinnen und Patienten, gleichzeitig soll es aber auch dem Gesundheitssystem, also den Kostenträgern und den Steakholdern, Rechnung tragen. Ein zunehmend unauflösliches Dilemma, das persönliche Wertesysteme in Spannung hält.

Ihre Zuspitzung erfuhr diese bekannte und dem Gesundheitssystem immanente Problematik in den 1980er Jahren, als Ökonomen begannen, monetäres Denken und Handeln in die Infrastruktur und Philosophie von Krankenhäusern und damit letztlich in die Patientenversorgung $\mathrm{zu}$ implementieren. Seit 2004, mit der Abkehr der Finanzierung über Tagessätze in deutschen Krankenhäusern, wird nicht nur jeder medizinische Fall anhand des DRG-Systems (Diagnosis Related Groups) pauschal vergütet, sondern vor allem wird jede Sparmaßnahme ebenso pauschal belohnt. Die komplexe Erlösstruktur von Krankenhäusern wird mit dieser Vorgehensweise vollständig einer sich kontinuierlich steigernden Gewinnmaximierung untergeordnet. Der Schlüsselfaktor hierbei ist die stationäre Verweildauer, welche einen sehr engen und stetig abnehmenden Korridor vorgibt, innerhalb dessen ein Krankenhaus die Behandlungen von stationären Fällen noch ohne Verluste abschließen kann. Die so fixierten und in vielen Fällen stark begrenzten, immer jedoch limitierten, finanziellen Mittel, welche nun abgekoppelt von der jeweils für den Patienten tatsächlich erbrachten Leistung zur Verfügung stehen, erhöhen den ökonomischen Druck auf die behandelnden Teams erheblich. Und dies, obwohl die ins Gesundheitssystem investierten Mittel nie größer waren als heute.

Hinzu kommt, dass sich im Arzt-Patienten-Verhältnis die Patienten in einer unterlegenen, asymmetrischen Beziehung befinden, welche eine besondere Schutzbedürftigkeit mit sich bringt, und daher eine gesteigerte ärztliche Fürsorge und verantwortliches Handeln erfordert, welches strukturell verankert und abgesichert sein sollte [1]. Zugleich steht die Ärzteschaft aber auch in der gesellschaftlichen Verantwortung, mit den begrenzten Ressourcen des Gesundheitssystems verantwortungsvoll umzugehen $[2,3]$.

In diesem Spannungsfeld ärztliches Ethos und wirtschaftlich verantwortungsbewusstes Handeln zu verbinden, fällt vor allem jungen MedizinerInnen zunehmend schwer. Im Rahmen des Medizinstudiums werden die Grundlagen zum gesundheitspolitischen und betriebswirtschaftlichen Wissen als Bestandteil des Querschnittsfachs „Gesundheitsökonomie, Gesundheitssystem, öffentliche Gesundheitspflege“ gelehrt, und sollen mit durchschnittlich einer Semes- terwochenstunde Vorlesung sowie 0,5 Semesterwochenstunden Seminar abgedeckt werden [4]. Dies ist ein knapp bemessenes Zeitfenster, sofern nicht nur ein rein ökonomisches Denken erlernt werden soll, sondern darüber hinaus ökonomie-kritische Reflektionen erfolgen sollen, oder gar angestrebt wird, ein auf Werthaltungen fußendes und ethisch-moralisches Selbstverständnis zu entwickeln.

Vorteilhaft für eigene Reflektionen sind die Kenntnisse der Strukturiertheit und Wirkweise individueller Werthaltungen auf Handlungsmotivationen und damit auch auf medizinische Verhaltensweisen. Der grundlegende Wirkzusammenhang zwischen individuellen Werthaltungen und einem daraus folgenden Handeln wurde von Rokeach bereits im Jahre 1968 beschrieben, wobei die Werte damals noch in Rangreihen geordnet wurden. Individuelle Werthaltungen werden heutzutage in Form des Wertekreises wiedergegeben, welcher von Bilsky und Schwartz Ende der 1980er entwickelt wurde, und hier in der modifizierten Version mit vier Segmenten Anwendung findet. Zur Universalität des Wertekreises führen Strack, Gennerich und Hopf [5, S. $101 \mathrm{f}$.] aus, dass sie „den Wertekreis für ein Inhaltsmodell einer Generalität, [halten] wie sie sonst nur [...], die Big Five [...] aufweisen“. Gestützt wird diese Auffassung durch Reanalysen der Wertedaten, welche im Rahmen einer kontinuierlichen und repräsentativen europäischen Sozialbefragung (European Social Survey) erhoben wurden.

\section{Zu dieser Studie}

Das in dieser Studie aufgegriffene Dilemma zwischen medizin-ethischen und ökonomischen Entscheidungskriterien ist geeignet, grundlegendes Misstrauen in der Bevölkerung, aber auch bei medizinischem Fachpersonal, zu erzeugen. Je geringer das Vertrauen in die Patientengesundheit als oberstes Entscheidungskriterium medizinischen Handelns ist, desto geringer lässt sich eine Compliance $\mathrm{zu}$ diesem Gebot bei medizinischem Fachpersonal erwarten.

In der hier vorliegenden quantitativen Erhebung soll eruiert werden, wie hoch inzwischen das Misstrauen in solche Entscheidungen bei Studierenden und medizinischem Personal ist, und ob sich bei Letztgenannten Unterschiede zwischen den jeweiligen Hierarchiestufen zeigen. Grundlage hierzu bilden die Antworten auf eine Vignette zu einer Entlassungsentscheidung, bei der die jeweiligen individuellen Werthaltungen der Befragten berücksichtigt werden.

\section{Material und Methoden}

\section{Struktur und Hypothesen}

Teilnehmende der vorliegenden Studie waren Ärztinnen und Ärzte in unterschiedlichen Positionen (Chefarzt/ärztin, Leitende/r Oberarzt/ärztin, Ober- 


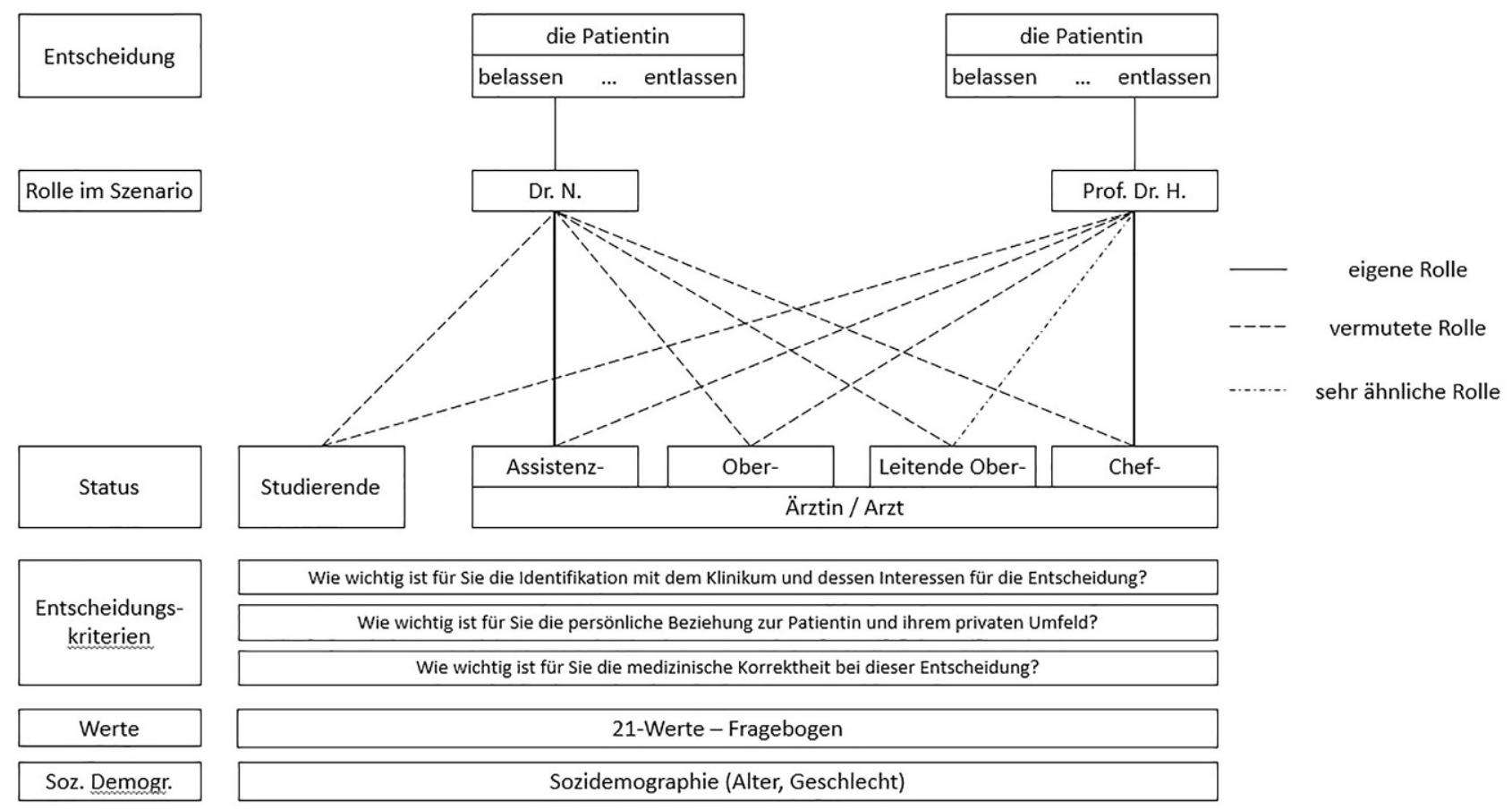

Abb. 1 Schema des Untersuchungsaufbaus

arzt/ärztin, Assistenzarzt/ärztin) sowie Medizinstudierende.

Die Studie war eine Querschnittsuntersuchung, welche über einen anonymen Fragebogen in Paper- \& Pencil-Form durchgeführt wurde. Ein Schema des Untersuchungsaufbaus ist der Abb. $1 \mathrm{zu}$ entnehmen; der Fragebogen findet sich im Anhang (Zusatzmaterial online).

Den Teilnehmenden wurde zunächst ein etabliertes Entscheidungsszenario des Institutes für Ethik und Geschichte der Medizin der Universitätsmedizin Göttingen vorgelegt, welches bei Garlichs entnommen wurde [6, S. $119 \mathrm{f}$.].

Das Setting dieses Szenarios erforderte eine Entscheidung über die Verweildauer einer älteren, noch nicht gänzlich genesenen Patientin, bei noch nicht geklärter häuslicher Weiterversorgung. Es bestanden die Entscheidungsalternativen, die Patientin in der Klinik zu belassen (Fürsorge) versus die Patientin aus der Klinik entlassen (Ökonomie), welche in einem 6-stufigen Rating differenziert werden konnten.

Diese Entscheidung sollten die Teilnehmenden einmal in der Rolle eines Arztes der Allgemeinchirurgie und einmal in der Rolle des Chefarztes treffen. Hier wurde somit ein klassischer Perspektivitätswechsel vorgenommen.

Erwartet wurde, dass an Stelle des Chefarztes Prof. H. weniger fürsorglich, sondern ökonomieorientierter als an Stelle des Dr. N. entschieden werden würde. Ein solcher Befund würde auf Misstrauen hindeuten.

Die Beantwortung des Fragebogens durch Assistenzärzte an Stelle von Dr. N., sowie durch Chefärzte und Leitende Oberärzte an Stelle von Prof. H., spiegelt die Entscheidung in der eigenen Position wider. Von besonderem Interesse ist hier, ob Chefärzte und Leitende Oberärzte tatsächlich ökonomieorientierter entscheiden.

Die Entscheidungen von allen anderen Teilnehmenden geben Vermutungen über die Rolle eines Assistenzarztes oder Chefarztes wieder. Misstrauen würde sich in einer stärkeren Befürwortung der Entlassung an Stelle des Chefarztes Prof. H. zeigen.

Nach der Be-/Entlassungsentscheidung aus den zwei unterschiedlichen Perspektiven wurden die Teilnehmenden gebeten, die Wichtigkeit der Entscheidungskriterien medizinischer Richtigkeit, Einfühlung in die Patientin, sowie die Identifikation mit dem Klinikum und dessen Interessen anzugeben, wobei hier ebenfalls eine Differenzierung über ein 6-stufiges Rating von „sehr wichtig“ bis „überhaupt nicht wichtig“ möglich war. Auch hier wurde erwartet, dass mit der Höhe der Position die Wichtigkeit des Klinikinteresses zuungunsten des Patientinneninteresses steigen würde.

Der dritte Abschnitt des Fragebogens enthielt die Soziodemographie.

Im letzten Abschnitt wurden die Teilnehmenden $\mathrm{zu}$ ihren individuellen Werthaltungen mittels eines Wertefragebogen befragt, welcher auf den Personal Value Questionnaire 21 (PVQ21) von Schwartz zurückgeht [7]. Dieser Wertefragebogen gewährleistet einerseits eine gute europäische Vergleichbarkeit der Befunde, da er im Rahmen der European Social Survey (ESS) seit 2001 alle 2 Jahre erhoben wird. Andererseits wird die hohe wissenschaftliche Qualität durch die betei- 
Tab. 1 Soziodemographische Daten der Teilnehmenden

\begin{tabular}{ll|l|l|l}
$\begin{array}{l}\text { Alter } \\
\text { Status }\end{array}$ & $\begin{array}{l}\text { Mittelwert } \\
\text { Jahre }\end{array}$ & $\begin{array}{l}\text { Minimum } \\
\text { Jahre }\end{array}$ & $\begin{array}{l}\text { Maximum } \\
\text { Jahre }\end{array}$ & Standardabweichung \\
\hline Alle Teilnehmenden & 30,37 & 19 & 66 & 9,449 \\
\hline Studierende & 24,54 & 19 & 46 & 3,855 \\
\hline Ärztinnen/Ärzte & 38,05 & 26 & 66 & 9,140 \\
\hline Assistenz-Ärztinnen/-Ärzte & 33,67 & 26 & 62 & 5,710 \\
\hline Ober-Ärztinnen/-Ärzte & 43,74 & 31 & 66 & 7,884 \\
\hline Leitende Ober-Ärztinnen/-Ärzte & 51,47 & 38 & 65 & 7,986 \\
\hline Chef-Ärztinnen/-Ärzte & 53,53 & 44 & 65 & 6,812 \\
\hline
\end{tabular}

Tab. 2 Soziodemographische Daten der Teilnehmenden

\begin{tabular}{|c|c|c|c|c|c|c|c|c|c|c|c|c|c|c|}
\hline \multirow{2}{*}{$\begin{array}{l}\text { Geschlecht } \\
\text { Status }\end{array}$} & \multicolumn{2}{|c|}{$\begin{array}{l}\text { Alle Teilnehmen- } \\
\text { den }\end{array}$} & \multicolumn{2}{|c|}{ Weiblich } & \multicolumn{2}{|c|}{ Männlich } & \multicolumn{2}{|c|}{ Divers } & \multicolumn{2}{|c|}{ Weiblich + Divers } & \multicolumn{2}{|c|}{ Keine Angaben } & \multicolumn{2}{|c|}{ Fehlt } \\
\hline & $N$ & $\%$ & $N$ & $\%$ & $N$ & $\%$ & $N$ & $\%$ & $N$ & $\%$ & $N$ & $\%$ & & \\
\hline Studierende & 684 & 55,2 & 473 & 61,8 & 207 & 44,4 & 0 & 0,0 & 1 & 100,0 & 3 & 60,0 & 0 & 0 \\
\hline Ärztinnen/Ärzte & 551 & 44,5 & 291 & 38,0 & 257 & 55,2 & 1 & 100,0 & 0 & 0,0 & 2 & 40,0 & 0 & 0 \\
\hline $\begin{array}{l}\text { Vermutlich Ärztinnen/Ärzte (da } \\
\text { Versorgungsstufe des Kranken- } \\
\text { hauses angegeben) }\end{array}$ & 3 & 0,2 & 1 & 0,1 & 2 & 0,4 & 0 & 0,0 & 0 & 0,0 & 0 & 0,0 & 0 & 0 \\
\hline Status + Genus fehlen & 1 & 0,1 & 0 & 0,0 & 0 & 0,0 & 0 & 0,0 & 0 & 0,0 & 0 & 0,0 & 1 & 100 \\
\hline Summe & 1239 & 100,0 & 765 & 100,0 & 466 & 100,0 & 1 & 100,0 & 1 & 100,0 & 5 & 100,0 & 1 & 100,0 \\
\hline
\end{tabular}

ligten Institutionen sichergestellt, da für Deutschland die ESS-Studien mit den Wertefragen durch das Leibniz-Institut für Sozialwissenschaften (GESIS) und die Deutsche Forschungsgemeinschaft (DFG) verantwortet werden. In Österreich erfolgt die Qualitätssicherung der ESS-Studien durch das Institut für höhere Studien - Institute for Advanced Studies, Vienna (IHS).

\section{Durchführung und statistische Auswertung}

Der Ethikantrag für die Studie wurde durch die EthikKommission der Medizinischen Fakultät der Christian-Albrechts-Universität zu Kiel gebilligt (Ethikvotum: 570/17) und die Studie gemäß den ethischen Grundsätzen der „Deklaration von Helsinki“ durchgeführt.

Befragt wurden im Zeitraum von Dezember 2017 bis April 2018 zwei Hauptgruppen: zum einen:

a) Studierende der Human- und Zahnmedizin an unterschiedlichen Universitäten Deutschlands vom 1.-12. Fachsemester mit der Angabe ihres angestrebten Fachbereichs, und zum anderen

b) Ärzte und Ärztinnen in unterschiedlichen Positionen (Assistenzarzt/ärztin, Oberarzt/ärztin, Leiten$\mathrm{de} / \mathrm{r}$ Oberärztin/arzt, Chefarzt/ärztin), welche in Krankenhäusern aller Versorgungsstufen (Grund-, Regel-, Schwerpunkt- und Maximalversorgung) tätig waren.

Die Teilnehmenden wurden in Vorlesungen, Seminaren, Frühbesprechungen und Klinikkonferenzen gewonnen.

Die Auswertung erfolgte in Microsoft-Excel@ und IBM-SPSS-25@.
Zur Bewertung der Effektstärken $d$ und Beta (übertragen aus $r$ ) wurden die Konventionen von Cohen (1988) zugrunde gelegt.

\section{Ergebnisse}

\section{Teilnehmende, Soziodemographie und Werthaltungen}

An der Befragung haben 1239 Personen teilgenommen, davon waren 44,5\% ÄrztInnen (Durchschnittsalter $=38,0$ Jahre) und 55,2\% Studierende (Durchschnittsalter $=24,6$ Jahre), weitere soziodemographische Daten sind in Tab. 1, 2, 3 und 4 wiedergegeben.

Die individuellen Werthaltungen der jeweiligen Teilnehmenden mit ihrer Verortung im Wertekreis sind in Abb. 2 wiedergegeben. Die Werthaltungsanalysen zeigen zwei korrelative Zusammenhänge. Zum einen zwischen den persönlichen Werthaltungen und dem Geschlecht, denn die teilnehmenden Frauen äußern im Vergleich zu Männern universalistischere Werte. Zum anderen besteht ein korrelativer Zusammenhang zwischen den persönlichen Werten und dem Status, denn die Studierenden geben im Vergleich zu den ÄrztInnen tolerantere, freiheitsliebendere Werte an (Abb. 2).

\section{Entscheidungskriterien}

Alle Teilnehmenden stellen in ihren Entscheidungen, ob die Patientin aus dem Krankenhaus entlassen werden soll oder noch nicht, die medizinische Korrektheit der Entscheidung in den Vordergrund $(\mathrm{MW}=1,26$; 
Tab. 3 Soziodemographische Daten der Teilnehmenden

\begin{tabular}{|c|c|c|c|c|c|c|c|c|c|c|c|c|}
\hline \multirow{2}{*}{$\begin{array}{l}\text { Geschlecht } \\
\text { Status }\end{array}$} & \multicolumn{2}{|c|}{ Studierende } & \multicolumn{2}{|c|}{ Studentinnen } & \multicolumn{2}{|c|}{ Studenten } & \multicolumn{2}{|c|}{ Divers Studierende } & \multicolumn{2}{|c|}{$\begin{array}{l}\text { Weiblich + Divers Studie- } \\
\text { rende }\end{array}$} & \multicolumn{2}{|c|}{ Keine Angaben } \\
\hline & $N$ & $\%$ & $N$ & $\%$ & $N$ & $\%$ & $N$ & $\%$ & $N$ & $\%$ & $N$ & $\%$ \\
\hline Studierende Studienfach offen & 353 & 51,6 & 248 & 52,4 & 102 & 49,3 & - & - & 0 & 0,0 & 3 & 100,0 \\
\hline Studierende mit Studienfach & 322 & 47,1 & 219 & 46,3 & 102 & 49,3 & - & - & 1 & 100,0 & 0 & 0,0 \\
\hline $\begin{array}{l}\text { Studierende mit Studienfach } \\
\text { und Studienfach offen ange- } \\
\text { kreuzt }\end{array}$ & 9 & 1,3 & 6 & 1,3 & 3 & 1,4 & - & - & 0 & 0,0 & 0 & 0,0 \\
\hline Summe & 684 & 100,0 & 473 & 100,0 & 207 & 100,0 & - & - & 1 & 100,0 & 3 & 100,0 \\
\hline
\end{tabular}

Tab. 4 Soziodemographische Daten der Teilnehmenden

\begin{tabular}{|c|c|c|c|c|c|c|c|c|c|c|c|c|}
\hline \multirow{2}{*}{$\begin{array}{l}\text { Geschlecht } \\
\text { Status }\end{array}$} & \multicolumn{2}{|c|}{ Ärztinnen/Ärzte } & \multicolumn{2}{|c|}{ Ärztinnen } & \multicolumn{2}{|l|}{ Ärzte } & \multicolumn{2}{|c|}{$\begin{array}{l}\text { Divers Ärztinnen/ } \\
\text { Ärzte }\end{array}$} & \multicolumn{2}{|c|}{$\begin{array}{l}\text { Weiblich + Divers Ärztin- } \\
\text { nen/Ärzte }\end{array}$} & \multicolumn{2}{|c|}{ Keine Angaben } \\
\hline & $N$ & $\%$ & $N$ & $\%$ & $N$ & $\%$ & $N$ & $\%$ & $N$ & $\%$ & $N$ & $\%$ \\
\hline Assistenz-Ärztinnen/-Ärzte & 352 & 63,9 & 221 & 75,9 & 130 & 50,6 & 1 & 100,0 & - & - & 0 & 0,0 \\
\hline Ober-Ärztinnen/-Ärzte & 131 & 23,8 & 54 & 18,6 & 76 & 29,6 & 0 & 0,0 & - & - & 1 & 50,0 \\
\hline Leitende Ober-Ärztinnen/-Ärzte & 40 & 7,3 & 8 & 2,7 & 31 & 12,1 & 0 & 0,0 & - & - & 1 & 50,0 \\
\hline Chef-Ärztinnen/-Ärzte & 19 & 3,4 & 1 & 0,3 & 18 & 7,0 & 0 & 0,0 & - & - & 0 & 0,0 \\
\hline $\begin{array}{l}\text { Sonstige/Freie Ärztinnen/Ärzte/ } \\
\text { Zahnärztinnen/-ärzte/Labor }\end{array}$ & 7 & 1,3 & 6 & 2,1 & 1 & 0,4 & 0 & 0,0 & - & - & 0 & 0,0 \\
\hline $\begin{array}{l}\text { Ärztinnen/Ärzte ohne Hierar- } \\
\text { chie }\end{array}$ & 2 & 0,4 & 1 & 0,3 & 1 & 0,4 & 0 & 0,0 & - & - & 0 & 0,0 \\
\hline Summe & 551 & 100,0 & 291 & 100,0 & 257 & 100,0 & 1 & 100,0 & - & - & 2 & 100,0 \\
\hline
\end{tabular}

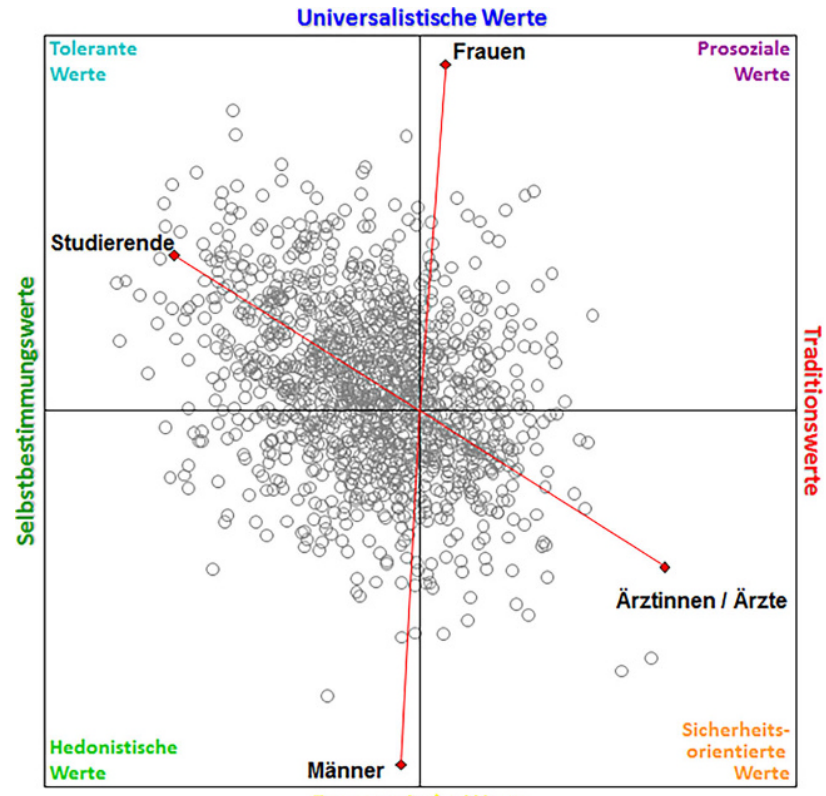

Abb. 2 Verteilung der individuellen Werthaltungen der Teilnehmenden im Wertekreis (inkl. Korrelationen mit Geschlecht und Status)

se $=0,02 ; 6$-stufige Skala mit $1=$ „sehr wichtig“ bis $6=$, überhaupt nicht wichtig“).

Eine geschlechtsspezifische Differenzierung, wie in Abb. 3 dargestellt, weist zwar aus, dass Ärztinnen die medizinische Korrektheit signifikant wichtiger ist $(\mathrm{MW}=1,22$; se $=0,03$ ) als Ärzten (MW=1,31; se =0,04), wobei dies jedoch nichts an der grundlegend sehr hohen Wichtigkeit der medizinisch fundierten Ent-

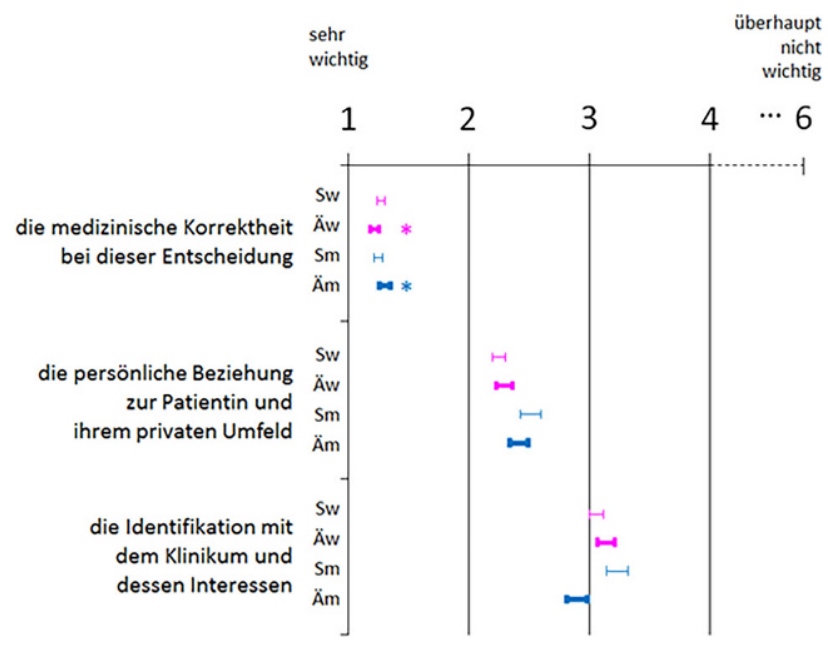

Abb. 3 Wichtigkeitsratings für die konfligierenden Entscheidungskriterien nach Status (S/Ä Studierende/ÄrztInnen) und Geschlecht $(\mathrm{w} / \mathrm{m})(\mathrm{MW} \pm 95 \% \mathrm{Cl})$

scheidungskorrektheit für die gesamte befragte ÄrztInnenschaft ändert.

Die Studierenden weisen hingegen keine geschlechtsspezifischen Unterschiede in der auch für sie sehr hohen Wichtigkeit der medizinischen Korrektheit der Entscheidung auf.

Neben der medizinischen Korrektheit wird von allen Teilnehmenden das Patientenwohl, also die persönliche Beziehung zur Patientin und ihrem privaten Umfeld, als ebenfalls wichtiges Entscheidungskriterium bewertet $(\mathrm{MW}=2,35$; se $=0,03 ; 6$-stufige Skala mit $1=$,sehr wichtig“ bis $6=$ „überhaupt nicht wich- 


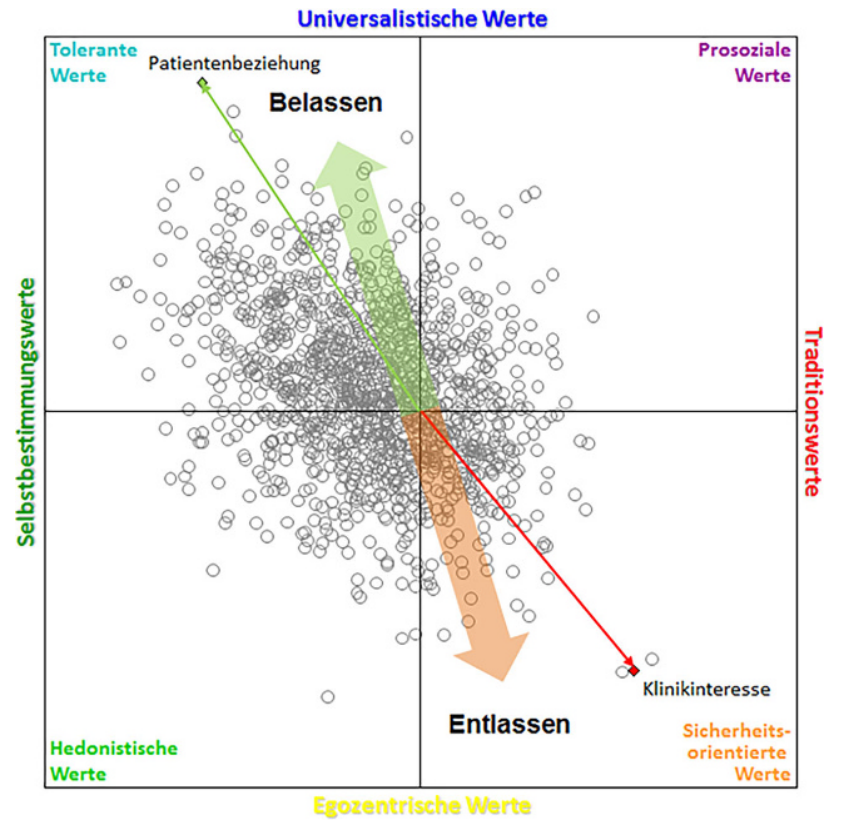

Abb. 4 Abhängigkeit der Entscheidungen und Entscheidungskriterien von den individuellen Werthaltungen der Teilnehmenden im Wertekreis

tig“). Dabei ist Studentinnen und Ärztinnen die Beziehungsebene tendenziell, wenn auch nicht signifikant, wichtiger als ihren männlichen Kollegen, ein als CareEthik diskutiertes Phänomen.

Denjenigen Teilnehmenden, welche vorwiegend Tolerante Werte präferieren, ist das Kriterium des Patientenwohls signifikant wichtiger als denjenigen, welchen sicherheitsorientierte Werte wichtiger sind (Korrelation mit Universalismus $r=0,195$ und mit Selbstbestimmung $r=0,129 ; p<0,01$; Abb. 4).

Eine nachgeordnete Bedeutung für alle Teilnehmenden hat dagegen die Identifikation mit dem Klinikum selbst und dessen Interessen $((\mathrm{MW}=3,08$; se $=0,04)$, etwa auf der Skalenmitte), wobei sie männlichen Ärzten wichtiger ist, d.h. die Interaktion Geschlecht $\times$ Status wird statistisch signifikant. Auch die Personengruppe mit vorwiegend sicherheitsorientierten Werten hält die Interessen der Klinik für wichtiger (Korrelation mit egozentrischen Werten $r=0,153$ und mit Traditionswerten $r=0,126 ; p<0,01 ;$ Abb. 4).

\section{Be-/Entlassungsentscheidung}

Dass die Patientin des Szenarios grundsätzlich nicht entlassen werden sollte, scheint über alle Teilnehmendengruppen hinweg Konsens zu sein (MW=1,69; $\mathrm{SD}=0,80$ mit $1=$ belassen und $6=$ entlassen).

Erwartungskonform zeigt sich jedoch, dass alle Teilnehmenden unterstellen, dass bei einer Entscheidung durch einen Stationsarzt für die Patientin eine höhere Sicherheit für das Verbleiben in der Klinik besteht $(\mathrm{MW}=1,48)$, als bei einer Entscheidung durch einen Chefarzt $(M W=1,90)$. Dieser unterstellte Unterschied ist signifikant mit einer Effektstärke nach Cohen von $\mathrm{d}_{\mathrm{w}}=0,37$.

Interessanterweise sind insbesondere Studierende an Stelle des Chefarztes weniger für den Verbleib der Patientin in der Klinik (MW=2,46), als an Stelle des Arztes $\left(\mathrm{MW}=1,59 ; p_{2 \mathrm{t}}=0,001\right)$. Indirekt drücken die Studierenden damit Misstrauen gegenüber Chefärzten aus.

Allerdings ist dieses Misstrauen hier unbegründet, denn die teilnehmenden statushohen ÄrztInnen (Leitende OberärztInnen + ChefärztInnen) belassen die Patientin tendenziell eindeutiger in der Klinik $(M W=1,35)$ als die teilnehmenden statusniedrigeren ÄrztInnen (Assistenz-ÄrztInnen) (MW=1,57; $p_{2 \mathrm{t}}=0,052$; Tab. 5).

Dementgegen würden die Studierenden (gemittelt über beide Rollen als Dr. N. und als Prof. H.) die Patientin sogar signifikant eher entlassen $(\mathrm{MW}=1,86)$ als die teilnehmenden Ärztinnen oder Ärzte ( $M W=1,46$; $\left.p_{2 \mathrm{t}}=0,001\right)$.

Ähnlich wie bei den Entscheidungskriterien zeigt sich auch in der Entlassungsentscheidung ein Geschlechtseffekt, da die Teilnehmerinnen sowohl in der Rolle von Dr. N. als auch in der Rolle von Prof. H. die Patientin eindeutiger in der Klinik $\left(\mathrm{MW}_{\text {weiblich }}=1,57\right.$; $\mathrm{MW}_{\text {männlich }}=1,75 ; p_{2 \mathrm{t}}=0,003$ ) belassen.

Die individuellen Werthaltungen der Teilnehmenden haben erwartungskonform ebenfalls einen Einfluss auf die Entlassungsentscheidung. Je prosozialer und toleranter, also zusammengenommen je universalistischer die Werthaltungen sind, desto eindeutiger wird die Patientin in der Klinik belassen.

\section{Diskussion}

Gesellschaftspolitisch sind heutzutage Krankenhäuser mehr denn je nicht nur dem medizinischen, sondern auch dem wirtschaftlichen Erfolg verpflichtet. Insofern ist es nur konsequent und folgerichtig, dass inzwischen deutsche Kliniken mehrheitlich von ÖkonomInnen geleitet werden. Allein durch diese Strukturveränderungen rücken wirtschaftliche Aspekte zunehmend in den Vordergrund, die zumindest indirekt Einfluss auf medizinische Entscheidungen nehmen können [8-11]. Darüber hinaus werden aber auch die medizinischen Leitungen der Kliniken in dieses System der Ökonomisierung eingebunden. Ärztinnen/ Ärzte in leitenden Funktionen, allen voran Chefärztinnen/Chefärzte, finden heutzutage ihre Dienstverträge oftmals derart gestaltet, dass neben einem Festgehalt eine monetäre Beteiligung am wirtschaftlichen Erfolg der Klinik vorgesehen ist.

Aus dieser Entwicklung erwächst jedoch unweigerlich ein Dilemma, konkurrieren doch die eigenen ökonomischen Interessen oft mit der Prämisse, dem Wohl der Patientin/des Patienten zu dienen. Insofern scheint die Befürchtung nicht gänzlich unbegründet $\mathrm{zu}$ sein, dass auf individueller Ebene die medizinische Unabhängigkeit zuungunsten des PatientIn- 
Tab. 5 Entlassungstendenz aus beiden Perspektiven (gemittelt), sowie als Chefarzt Prof. H. vs. Arzt Dr. N. (Geschätzte MW und $p$ aus einem mehrfaktoriellen Modell)

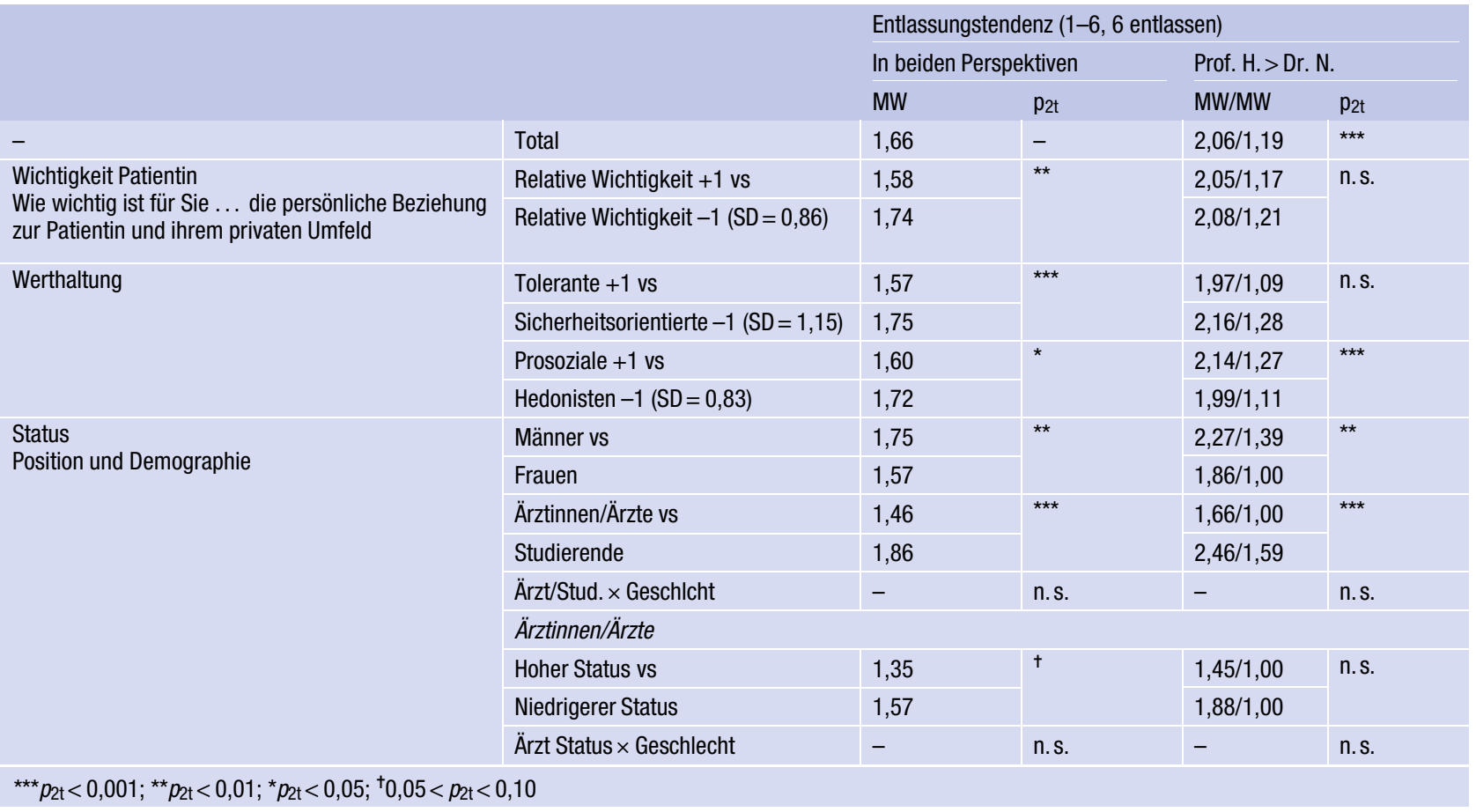

nenwohls gefährdet sein könnte [12-14]. Unstrittig ist zumindest, dass der stetig wachsende finanzielle Druck zwischenzeitlich von allen Beteiligten beobachtet wird. Die deutlich gesteigerte Wahrnehmung, dass wirtschaftliche Interessen das Handeln von AkteurInnen in Krankenhäusern beeinflussen könnten, haben Wehkamp und Naegler an Hand von Interviews sowohl mit Geschäftsführungen von Krankenhäusern als auch mit Ärztinnen/Ärzten analysiert. In ihrer qualitativen Studie berichtet insbesondere die Gruppe der Ärztinnen/Ärzte von strategischen Patientenaufnahmen, aber auch von konkreten Beeinflussungen der Verweildauer [15].

Dem ökonomischen Druck steht entgegen, dass die ärztliche Berufsordnung ( $§$ 2) explizit festlegt, dass „Ärztinnen und Ärzte ihren Beruf nach ihrem Gewissen, den Geboten der ärztlichen Ethik und der Menschlichkeit“ ausüben sollen. „Sie dürfen keine Grundsätze anerkennen und keine Vorschriften oder Anweisungen beachten, die mit ihren Aufgaben nicht vereinbar sind oder deren Befolgung sie nicht verantworten können.“ Darüber hinaus haben sie „dabei ihr ärztliches Handeln am Wohl der Patientinnen und Patienten auszurichten. Insbesondere dürfen sie nicht das Interesse Dritter über das Wohl der Patientinnen und Patienten stellen." Hierzu gehört ganz dezidiert, dass Weisungen von Nichtärztinnen/Nichtärzten (z. B. Verwaltungsangestellte) weder entgegengenommen noch befolgt werden dürfen [16].

In der vorliegenden Vignetten-Studie ist untersucht worden, inwiefern unterschiedliche ärztliche Statusgruppen und Medizinstudierende glauben, dass der wachsende ökonomische Druck die Entlassung einer Patientin beeinflussen kann. Grundlegend beruhigend für alle Patientinnen und Patienten kann konstatiert werden, dass die Patientin der Vignette sowohl von allen Statusgruppen der Ärztinnen und Ärzte als auch von den Studierenden weiterhin in der Klinik belassen worden wäre. Das hierfür ausschlaggebende Entscheidungskriterium war für alle Teilnehmenden gleichermaßen das Primat ärztlichen Handelns, sich ausschließlich an medizinischen Kriterien zu orientierten, gerade so, wie es die ärztlichen Standesregeln vorsehen [16].

Nur in der Deutlichkeit der Entscheidung oder der graduellen Wichtigkeit von Entscheidungskriterien kommen individuelle Werthaltungen oder die Zugehörigkeit zu Statusgruppen des Medizinsystems zum Tragen. Dass Werthaltungen von Frauen etwas universalistischer als die von Männern sind, ist auch aus dem European Social Survey (ESS) und anderen repräsentativen Studien bekannt [17]. Darüber ist den freiheitsliebenden und toleranzorientierten Teilnehmenden das persönliche Wohl der Patientin der Vignette noch wichtiger als den sicherheitsorientierten Teilnehmenden. Beide Ergebnisse korrespondieren mit dem medizinethischen Ansatz „Care versus Justice“ von Gilligan [18, 19]. Grundsätzlich würden Personen mit universalistischen und Toleranz-Werten die Patientin am eindeutigsten in der Klinik belassen. Allerdings sind Studierende, die ja freiheitsliebende und toleranzorientiertere Werthaltungen angegeben haben, in ihrer Entscheidung (an Stelle eines Arztes oder gar Chefarztes) unsicherer als ÄrztInnen selbst. 
Innerhalb der Ärzteschaft fällt die Entscheidung zugunsten des Verbleibs der Patientin in der Klinik mit zunehmend höherem Status deutlicher und damit sicherer aus.

Einer eingehenderen Thematisierung bedarf es allerdings, dass die Teilnehmenden der Studie grundsätzlich dem Chefarzt Prof. H. unterstellt haben, dass er die Patientin eher entlassen würde, er sich also möglicherweise stärker den (finanziellen) Klinikinteressen verpflichtet fühlt. Dieses Misstrauen wurde umso größer, je prosozialer die Werthaltungen der Teilnehmenden waren. Bei den Studierenden war dieses größer ist als bei den ÄrztInnen. In der Studie erwies sich dieses Misstrauen jedoch als inakkurat, da sich die teilnehmenden Leitenden OberärztInnen und ChefärztInnen eindeutig für einen Verbleib der Patientin in der Klinik aussprachen.

Jedoch allein schon die Vermutung über die möglichen Entscheidungspräferenzen von Vorgesetzten, kann im Berufsleben zu Konsequenzen im eigenen Entscheidungsverhalten führen.

Andere empirische Untersuchungen belegten das Potential betriebswirtschaftlicher Vorgaben in Verträgen der GeschäftsführerInnen mit den Kliniken, die ärztlichen patientenbezogenen Entscheidungen $\mathrm{zu}$ beeinflussen $[8,14,20,21]$. So verneinten zwar die GeschäftsführerInnen in der Studie von Wehkamp und Naegler eine direkte Einflussnahme auf das ärztliche Handeln, aber einer mittelbaren Einflussnahme auf Grund der ökonomischen Vorgaben wird durchaus zugestimmt [15]. Die Diskrepanz zwischen betriebswirtschaftlichen Vorgaben und der patientenbezogenen Entscheidungsautonomie wird in der Ärzteschaft durchaus als problematisch wahrgenommen. Allein die Vermutung einer möglicherweise ökonomisch motivierten Entscheidung des eigenen Vorgesetzten, und damit hat sich unsere Vignettenstudie ja eigentlich beschäftigt, birgt bereits das Risiko eines vorauseilenden Gehorsams in sich.

Hiergegen könnte auf individueller Ebene eine gestärkte und souveräne Entscheidungsautonomie immunisieren. Die Frage, ob diese jedoch durch die verschulte Ausbildungsform mit ihrem recht geringen Anteil medizinethischer Inhalte in ausreichendem Maße erfolgen kann, verbleibt zu klären.

Damit sich Ärzte und Ärztinnen im Laufe ihrer Aus- und Weiterbildung eine medizinethisch basierte, deontologisch fundierte Entscheidungsautonomie aneignen, die sich im Einzelfall auch aktuellen Regeln widersetzen kann, gerade wenn solche nicht der Berufsethik entsprechen, sollte bereits die universitäre Lehre zur Berufsethik und ihres deontologischen Hintergrunds ausgeweitet werden. Ein anderer, grundlegender Ansatz wäre eine ökonomische Neuausrichtung. Diese würde jedoch einen Umbau des inhärenten Systems bedingen und einen veränderten politischen Willen voraussetzen.

Die Rechte der Teilnehmenden wurden gewahrt. Studien an Tieren wurden nicht durchgeführt.
Danksagung Die Autoren danken Prof. Dr. Mark Schweda, Abteilung für Medizinische Ethik der Carl von Ossietzky Universität Oldenburg für seinen Beitrag in der Erarbeitung des klinischen Szenarios sowie allen teilnehmenden Studierenden und Ärztinnen/Ärzten für die Ermöglichung dieser Erhebung.

\section{Einhaltung ethischer Richtlinien}

Interessenkonflikt I. Alkatout, M. Strack, N. Maass, M. Boos und N. Hopf geben an, dass kein Interessenkonflikt besteht.

Ethische Standards Der Ethikantrag für die Studie wurde durch die Ethik-Kommission der Medizinischen Fakultät der Christian-Albrechts-Universität zu Kiel gebilligt (Ethikvotum: 570/17) und die Studie gemäß den ethischen Grundsätzen der „Deklaration von Helsinki“ durchgeführt. Die Rechte der Teilnehmenden wurden gewahrt. Studien an Tieren wurden nicht durchgeführt.

Open Access Dieser Artikel wird unter der Creative Commons Namensnennung 4.0 International Lizenz veröffentlicht, welche die Nutzung, Vervielfältigung, Bearbeitung, Verbreitung und Wiedergabe in jeglichem Medium und Format erlaubt, sofern Sie den/die ursprünglichen Autor(en) und die Quelle ordnungsgemäß nennen, einen Link zur Creative Commons Lizenz beifügen und angeben, ob Änderungen vorgenommen wurden.

Die in diesem Artikel enthaltenen Bilder und sonstiges Drittmaterial unterliegen ebenfalls der genannten Creative Commons Lizenz, sofern sich aus der Abbildungslegende nichts anderes ergibt. Sofern das betreffende Material nicht unter der genannten Creative Commons Lizenz steht und die betreffende Handlung nicht nach gesetzlichen Vorschriften erlaubt ist, ist für die oben aufgeführten Weiterverwendungen des Materials die Einwilligung des jeweiligen Rechteinhabers einzuholen.

Weitere Details zur Lizenz entnehmen Sie bitte der Lizenzinformation auf http://creativecommons.org/licenses/by/4. $0 /$ deed.de.

\section{Literatur}

1. Alkatout I. Communicative and ethical aspects of physician-patient relationship in extreme situations. Wien Medizinische Wochenschrift. 2015;165(23-24:491-8.

2. Heyers J. Prädiktive Gesundheitsinformationen - Persönlichkeitsrechte und Drittinteressen. Medizinrecht. 2009;27:507-12.

3. Flintrop J. Krankenhäuser zwischen Medizin und Ökonomie - Die Suche nach dem richtigen Maß. Dtsch Arztebl. 2014;111(45):A1929-A31.

4. Nationaler Kompetenzbasierter Lernzielkatalog Medizin (NKLM) [database on the Internet]. MFT Medizinischer Fakultätentag der Bundesrepublik Deutschland e.V. 2015. Available from: http://www.nklm.de/files/nklm_final_ 2015-07-03.pdf.

5. Strack M, Gennerich C, Warum Werte HN. In: Witte E Ed Sozialpsychologie Werte Lengerich: Pabst;.2008;p:90-130.

6. Garlichs E. Über die Motivation, einen helfenden Beruf anzustreben: eine Befragung von Pädagogik-, Psychologieund Medizinstudenten und -studentinnen. 1. Aufl. Konstanz: Hartung-Gorre; 2000.

7. ESS Source Questionnaire Available from: http://www. europeansocialsurvey.org/.Zugegriffen: 14.11.2019 
8. Baberg HT, Kielstein R, de Zeeuw J, Sass HM. Treatment regulations and treatment limits: factors influencing clinical decision-making. Dtsch Med Wochenschr. 2002;2;127(31-32):1633-7. Aug.

9. Alkatout I, Rummer A. Intrauterines Lebensrecht von Zwillingen mit ungleichen Überlebenschancen - Kommentar zum Fall. Ethik Medizin. 2011;23(3):233-4.

10. Bühmann F. Kliniken müssen Geld verdienen - Podiumsdiskussion bei. Schleswiger Nachrichten:Helios; 2015.

11. Kobberling J. Economic Pressure in Hospitals. Dtsch Arzteblatt Int. 2017;24;114(47):795-6. Nov.

12. Salomon F, Moral Abhängigkeit ZA. Moral und Abhängigkeit. Ethik Medizin. 2007;19(3):174-86.

13. AlkatoutI, Mettler L, Maass N, AckermannJ. Robotic surgery in gynecology. J Turk Ger Gynecol Assoc. 2016;17(4):224-32.

14. Schumm-Draeger PM, Mann K, Muller-Wieland D, Folsch UR. The Patient is not a customer and the hospital not a business center. Dtsch Med Wochenschr. 2016;141(16):1183-5.Aug.

15. Wehkamp KH, Naegler H. The Commercialization of Patient-Related Decision Making in Hospitals. Dtsch ArzteblattInt. 2017;24;114(47):797-804. Nov.

16. Muster-Berufsordnung für die in Deutschland tätigen Ärztinnen und Ärzte [database on the Internet]. Deutsches
Ärzteblatt. 2015 [cited Letzter Zugriff: 16. November 2019]. Available from: http://www.bundesaerztekammer.de/ fileadmin/user_upload/downloads/pdf-Ordner/MBO/ MBO_2.Juli2015.pdf.

17. Schwartz SH, Rubel T. Sex differences in value priorities: cross-cultural and multimethod studies. J Pers Soc Psychol. 2005;89(6):1010-28. Dec.

18. Gilligan C, Attanucci J. The Moral Principle of Care. Introducing Psychological Research. London: Palgrave; 1996.

19. Gilligan C, Hrsg. In a different voice: psychological theory and women's development. Cambridge, Massachusetts: Harvard University Press; 1982.

20. Vreeke G. Gilligan on Justice and Care: two interpretations. JMoral Educ. 1991;20(1):33-46.

21. NoackT., Fangerau, H.ZurGeschichte des Verhältnissesvon Arzt und Patient in Deutschland. In Schulz S, Steigleder K, Fangerau H, Paul NW (Hrsg) Geschichte, Theorie und Ethik der Medizin-Eine Einführung. Frankfurt: Suhrkamp; 2006. S.77-93.

Hinweis des Verlags Der Verlag bleibt in Hinblick auf geografische Zuordnungen und Gebietsbezeichnungen in veröffentlichten Karten und Institutsadressen neutral. 